Copyright by the Acoustical Society of America. Rossi, A., Liang, C., \& Rogers, C. A. (1994). Dynamic analysis of piezoelectric actuator-driven circular rings using an impedance approach. Journal of the Acoustical Society of America, 96(3), 1592-1597. doi: 10.1121/1.410237

\title{
Dynamic analysis of piezoelectric actuator-driven circular rings using an impedance approach
}

\author{
Anna Rossi \\ Aerospace Department, University of Rome, Rome, Italy \\ Chen Liang and Craig A. Rogers \\ Center for Intelligent Material Systems and Structures, Virginia Polytechnic Institute and State University, \\ Blacksburg, Virginia 24061-0261
}

(Received 5 February 1993; revised 28 February 1994; accepted 2 May 1994)

This paper presents a dynamic model for the response of a circular ring excited by piezoelectric transducer (PZT) actuators bonded on the ring surface. The dynamic response is determined based on the dynamic interaction between the PZT actuators and the structure using an impedance approach. Compared with the conventional static approach, in which a statically determined "equivalent force" of the actuator is used as the forcing function in the dynamic analysis, the impedance approach cannot only capture the physics of the actuator/structure interaction, but also accurately predict the structural dynamic response. Experiments have also been conducted to verify the theoretical model. The predicted dynamic response using the impedance approach agrees very well with the experimental results. Comparison of the conventional static approach and the impedance model has also been presented.

PACS numbers: $43.40 . \mathrm{Vn}$

\section{INTRODUCTION}

Piezoceramics have often been used as actuators for active structural vibration and acoustic control. The structural behavior (for beams and plates) under the excitation of piezoceramic actuators was modeled by Crawley and de Luis (1987) and Dimitriadis et al. (1989). Most dynamic analyses of adaptive structures with integrated actuators have been based on a static approach, namely, using a statically determined transmitting force as the forcing function in the dynamic analysis.

Liang et al. (1993) have developed an impedance modeling methodology for the dynamic analysis of adaptive material systems. Unlike the conventional static approach, the impedance approach determines the actuator transmitting force based on actuator and structural impedance, rather than on the static actuator and structural stiffness. The transmitting force depends on the global structural dynamics, including boundary conditions, structural damping, stiffness, and mass. The dynamic response determined with the static approach is a scaled frequency response function (ratio of response to excitation force), not the true response under a variable force excitation.

This paper will apply the general impedance methodology to a circular ring. Experimental results will be presented to validate the theoretical model. Comparison between the impedance approach and the static approach will also be conducted.

\section{SYSTEM DYNAMIC MODELING}

Consider a ring or a cylinder for which the axial traveling wave is not considered. Two PZT actuators are bonded on the top and bottom of the ring surface to create a pure bending moment, as shown in Fig. 1. The ring is assumed to be "thin" so that the linear Love-Kirchhoff theory (Leissa,
1973; Soedel, 1981) can be used. The actuator is also assumed to be "very thin" so that the stress across the actuator thickness remains constant in order to use the impedance model. To simplify the derivation, the width of the ring is assumed to be unity.

Surface bonded PZT actuators (ideally bonded) interact with their substrate in the form of shear force. However, most of the shear force is concentrated at the end of actuators within two to three actuator thickness distance (Lin and Rogers, 1993). For most cases, the interaction of integrated induced strain actuator (embedded or bonded) and its substrate can be modeled as a concentrated force at the end of the actuator. The interaction of a pair of surface bonded PZT actuators activated out-of-phase may then be modeled as a pair of moments, as shown in Fig. 1. The two concentrated bending moments on the two ends of the actuator can be expressed with the Dirac function as

$$
M_{\text {right }}=F(h+s) \delta\left(\theta-\theta_{p}\right)
$$

and

$$
M_{\text {left }}=-F(h+s) \delta\left(\theta+\theta_{p}\right),
$$

where $F$ is the force exerted on the structure by a PZT actuator at its edge, and $s$ and $h$ are the actuator and ring thicknesses, respectively. Here, $\theta_{p}$ is the angular coordinate of the actuator edge, as shown in Fig. 1 . Note that $F$ is the dynamic transmitting force and is presently unknown. The transmitting force $F$, acting on the ring, also satisfies the following relation:

$$
Z=\frac{F}{\dot{v}_{\theta=\theta_{p}}}=\frac{F}{i \omega v_{\theta=\theta_{p}}},
$$

where $Z$ is the mechanical impedance of the ring at the actuator edge (ring/actuator interface) corresponding to a force, 


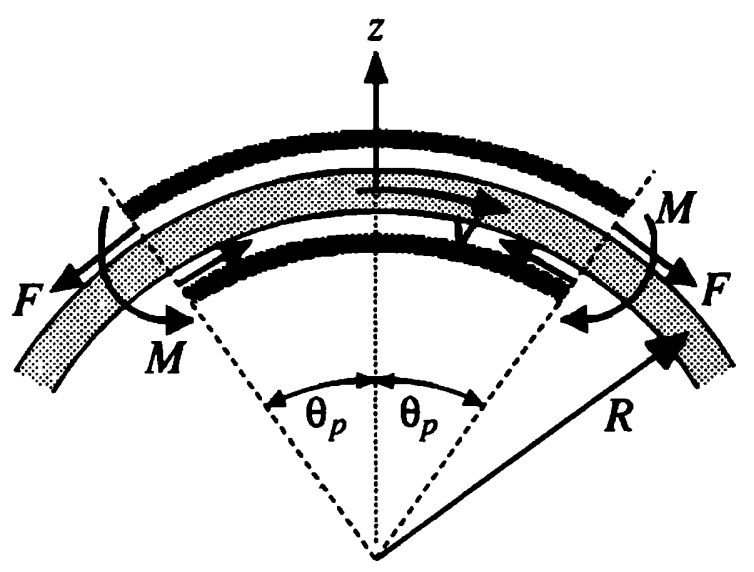

FIG. 1. One-dimensional circular ring with bonded PZT actuators activated out-of-phase.

$F$, in the circumferential direction, $v_{\theta=\theta p}$ is the circumferential displacement at the force location, and a dot indicates a function of time derivative.

One PZT actuator (top or bottom) and its interaction with the ring may be represented by a generic system as shown in Fig. 2. The curvature effect has not been considered in the dynamic modeling of the piezoelectric actuators. It is, therefore, necessary to assume that the patch's dimension be kept small relative to the cylinder radius so that the error due to the curvature effect can be neglected. The motion of the piezoelectric will be considered along the $d_{32}$ direction (or in the global $y$ direction).

As an electrical field is applied in the actuator thickness direction, the actuator expands and contracts in the $y$ direction $\left(d_{32}\right.$ effect). The one-dimensional constitutive equation for the actuator can be written as

$$
S_{2}=\bar{s}_{22}^{E} T_{2}+d_{32} E,
$$

where $S_{2}$ is the strain, $T_{2}$ the stress, $E$ the electric field, $d_{32}$ the piezoelectric coefficient, and $\bar{s}_{22}^{E}$ the complex compliance at a constant electrical field.

The equation of motion of the actuator vibrating in the $y$ direction may be expressed as follows:

$$
\rho_{p} \frac{\partial^{2} v}{\partial t^{2}}=\bar{Y}_{22}^{E} \frac{1}{R^{2}} \frac{\partial^{2} v}{\partial \theta^{2}},
$$

where $v$ is the displacement in the $y$ direction, $\rho_{p}$ is the density of the PZT actuator, $\bar{Y}_{22}^{E}$ is the complex modulus of the PZT at a constant electrical field and $R$ is the radius of the ring. The thickness of the ring and the PZT actuator is

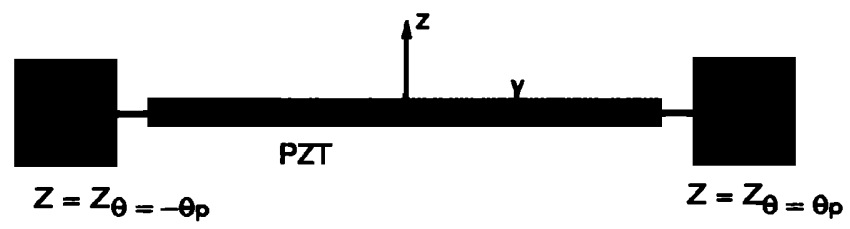

FIG. 2. A generic representation of the dynamic interaction of a surface bonded PZT actuator. much smaller than the radius and is ignored in the above equation.

Assuming a harmonic excitation and solving Eq. (5) by separating the displacement into time and spatial domain yields:

$$
v=\bar{v} e^{i \omega t}=[A \sin (k R \theta)+B \cos (k R \theta)] e^{i \omega t},
$$

where the wave number $k$ is given by

$$
k^{2}=\omega^{2}\left(\rho_{p} / \bar{Y}_{22}^{E}\right),
$$

where $\omega$ is the excitation frequency.

The boundary condition of the PZT actuator is illustrated in Fig. 2. The interaction between actuator and substructure is taken into account by the equilibrium and compatibility equations which are written in terms of the point impedance of the substructure:

$$
\left.T_{2}\right|_{\theta=\theta_{p}} s=-\left.\left.i \omega Z\right|_{\theta=\theta_{p}} v\right|_{\theta=\theta_{p}}
$$

and

$$
\left.T_{2}\right|_{\theta=-\theta_{p}} s=-\left.\left.i \omega Z\right|_{\theta=-\theta_{p}} v\right|_{\theta=-\theta_{p}} .
$$

The above equations describe the complex boundary conditions for the PZT actuator, which is given by transmitting force $=$ host structural impedance $x$ driving point velocity.

The two boundary conditions [Eqs. (8) and (9)], together with the constitutive equation of the PZT, provide the necessary conditions to determine $A$ and $B$. Substituting Eqs. (8) and (9) into Eq. (4), yields:

$$
\begin{aligned}
\left.\bar{S}_{22}\right|_{\theta=-\theta_{p}}= & \left.\frac{1}{R} \frac{\partial \nu}{\partial \theta}\right|_{\theta=-\theta_{p}}=-\frac{1}{\bar{Y}_{22}^{E}} \frac{\left.\left.Z\right|_{\theta=-\theta_{p}} v\right|_{\theta=-\theta_{p}} i \omega}{s} \\
& +d_{32} \bar{E}
\end{aligned}
$$

and

$\left.\bar{S}_{22}\right|_{\theta=\theta_{p}}=\left.\frac{1}{R} \frac{\partial \nu}{\partial \theta}\right|_{\theta=\theta_{p}}=-\frac{1}{\bar{Y}_{22}^{E}} \frac{\left.\left.Z\right|_{\theta=\theta_{p}} v\right|_{\theta=\theta_{p}} i \omega}{s}+d_{32} \bar{E}$,

where variables with a bar indicate spatial components except for $\bar{Y}_{22}^{E}$. Substituting Eq. (6) into Eqs. (10) and (11), a linear equation system in the unknowns $A$ and $B$ is obtained. Considering the symmetry of the ring (impedance at $-\theta_{p}$ and $\theta_{p}$ are the same and is denoted as $Z$ in the subsequent analysis), $B$ can be determined as zero and $A$ is given by

$$
A=\frac{d_{32} \bar{E}}{k \cos k R \theta_{p}+\left(Z i \omega / \bar{Y}_{22}^{E} s\right) \sin k R \theta_{p}} .
$$

The short-circuit actuator mechanical impedance (Liang et al., 1993) for a ring configuration may be derived as

$$
Z_{p}=\frac{\bar{Y}_{22}^{E} s}{R \theta_{p} i \omega} \frac{k R \theta_{p}}{\tan \left(k R \theta_{p}\right)}
$$

and the coefficient $A$ can be simplified as

$$
A=\frac{Z_{p} d_{32} \bar{E}}{\left(Z+Z_{p}\right) k \cos \left(k R \theta_{p}\right)} .
$$

The strain and stress within the actuator can be solved as 


$$
\bar{S}_{2}=\frac{Z_{p} d_{32} \bar{E}}{\left(Z+Z_{p}\right) \cos \left(k R \theta_{p}\right)} \cos (k R \theta)
$$

and

$$
\bar{T}_{2}=\left(\frac{Z_{p} d_{32} \bar{E}}{\left(Z+Z_{p}\right) \cos \left(k R \theta_{p}\right)} \cos (k R \theta)-d_{32} \bar{E}\right) \bar{Y}_{22}^{E} .
$$

The transmitting force between the actuator and host ring at $\theta_{p}$ can be determined as

$$
\left.F\right|_{\theta=\theta_{p}}=\left.\bar{T}_{2}\right|_{\theta=\theta_{p}} s=-\frac{Z d_{32} \bar{E} \bar{Y}_{22}^{E} s}{Z+Z_{p}} .
$$

It is clear that the transmitting force resulting from the activation of the PZT actuator is governed by the mechanical impedance of the host structure and the actuator. The induced moment of the actuator can then be determined from Eqs. (1), (2), and (17).

\section{DETERMINATION OF STRUCTURAL IMPEDANCE}

Equation (17) indicates that activation of a PZT actuator-results in a frequency-dependent excitation to the host structure. The transmitting force depends on the structural impedance. This section describes the derivation of structural impedance of the host ring corresponding to the loading of PZT actuator excitation. The impedance calculation will be performed only at position $\theta_{p}$ because of the symmetry of the ring structure. The equation of motion for the ring can be written in matrix form (Soedel, 1981) as

$$
\begin{aligned}
& \left\{\begin{array}{cc}
\frac{D}{R^{4}} \frac{d^{2}}{d \theta^{2}}+\frac{K}{R^{2}} \frac{d^{2}}{d \theta^{2}} & -\frac{D}{R^{4}} \frac{d^{3}}{d \theta^{3}}+\frac{K}{R^{2}} \frac{d}{d \theta} \\
\frac{D}{R^{4}} \frac{d^{3}}{d \theta^{3}}-\frac{K}{R^{2}} \frac{d}{d \theta} & -\frac{D}{R^{4}} \frac{d^{4}}{d \theta^{4}}-\frac{K}{R^{2}}
\end{array}\right\}\left(\begin{array}{l}
v \\
w
\end{array}\right)-\rho_{r} h\left(\begin{array}{l}
\ddot{v} \\
\ddot{w}
\end{array}\right) \\
& =\left(\begin{array}{c}
\frac{M_{\text {right }}}{R^{2}} \\
\frac{1}{R^{2}} \frac{d M_{\text {right }}}{d \theta}
\end{array}\right),
\end{aligned}
$$

where

$$
D=\frac{h^{3} \bar{Y}_{r}}{12\left(1-\mu^{2}\right)}
$$

and

$$
K=\frac{h \bar{Y}_{r}}{\left(1-\mu^{2}\right)} .
$$

Here, $\bar{Y}_{r}$ is the complex Young's modulus of the ring, $\rho_{r}$ the density of the ring, and $\mu$ the Poisson's ratio. The in-plane displacement $v$ and the transverse displacement $w$ may be expressed as

$$
v(t, \theta)=\sum_{n=1}^{\infty} v_{n}(t) \phi_{n}(\theta)
$$

and

$$
w(t, \theta)=\sum_{n=1}^{\infty} w_{n}(t) \psi_{n}(\theta) .
$$

For a one-dimensional ring, the eigenfunctions are $\phi_{n}(\theta)=\sin (n \theta)$ and $\psi_{n}(\theta)=\cos (n \theta)$. Substituting Eqs. (21) and (22) into Eq. (18) yields:

$$
\begin{aligned}
& \left\{\begin{array}{cc}
-\frac{D}{R^{4}} n^{2}-\frac{K}{R^{2}} n^{2} & -\frac{D}{R^{4}} n^{3}-\frac{K}{R^{2}} n \\
-\frac{D}{R^{4}} n^{3}-\frac{K}{R^{2}} n & -\frac{D}{R^{4}} n^{4}-\frac{K}{R^{2}}
\end{array}\right\}\left(\begin{array}{l}
v_{n} \\
w_{n}
\end{array}\right)-\rho_{r} h\left(\begin{array}{l}
\ddot{v}_{n} \\
\ddot{w}_{n}
\end{array}\right) \\
& =\left(\begin{array}{c}
\frac{M_{\text {right }} \sin (n \theta)}{\pi R^{2}} \\
\frac{M_{\text {right }} n \sin (n \theta)}{\pi R^{2}}
\end{array}\right) .
\end{aligned}
$$

To determine structural impedance, the transmitting force or the moment acting at $\theta_{p}$ is assumed to have a known magnitude. Assuming a harmonic excitation, the excitation moment may be expressed as $M_{\text {right }} e^{i \omega t}$. The modal amplitudes, $w_{n}(t)$ and $v_{n}(t)$, become

$$
w_{n}(t)=W_{n} e^{i \omega t}
$$

and

$$
v_{n}(t)=V_{n} e^{i \omega t} .
$$

Substituting Eqs. (24) and (25) into Eq. (23) yields a linear system of equations, from which the modal coefficients, $V_{n}$ and $W_{n}$, can be determined from the following equation:

$$
\begin{gathered}
\left\{\begin{array}{c}
-\frac{D}{R^{4}} n^{2}-\frac{K}{R^{2}} n^{2}+\rho_{r} h \omega^{2} \quad-\frac{D}{R^{4}} n^{3}-\frac{K}{R^{2}} n \\
-\frac{D}{R^{4}} n^{3}-\frac{K}{R^{2}} n \quad-\frac{D}{R^{4}} n^{4}-\frac{K}{R^{2}}+\rho_{r} h \omega^{2}
\end{array}\right\} \\
\times\left(\begin{array}{c}
V_{n} \\
W_{n}
\end{array}\right)=\left(\begin{array}{c}
\frac{M_{\text {right }} \sin (n \theta)}{\pi R^{2}} \\
\frac{M_{\text {right }} \sin (n \theta)}{\pi R^{2}}
\end{array}\right) .
\end{gathered}
$$

The point impedance of the ring can now be determined once the driving point velocity is determined. The driving point (surface of the ring) velocity includes the inplane displacement and the transverse bending, and may be expressed as

$$
\left.\dot{v}\right|_{\theta=\theta_{p}}=i \omega \sum_{n=1}^{\infty}\left(V_{n}+\frac{h}{2 R} n W_{n}\right) \sin (n \theta) \text {. }
$$

The driving point structural impedance from Eq. (3) can then be substituted into Eqs. (15) and (16) to determine the stress and strain within the PZT actuator. The induced moment of the actuators can be determined from Eqs. (1), (2), and (17).

When calculating the structural impedance, an arbitrary force (frequency independent) $\boldsymbol{F}$ may be used, which yields a structural response, $w(\theta, \omega)$ and $v(\theta, \omega)$. If a unit force is used in the calculation, the structural responses are actually the frequency response functions of the ring. If the frequency response functions are denoted as 


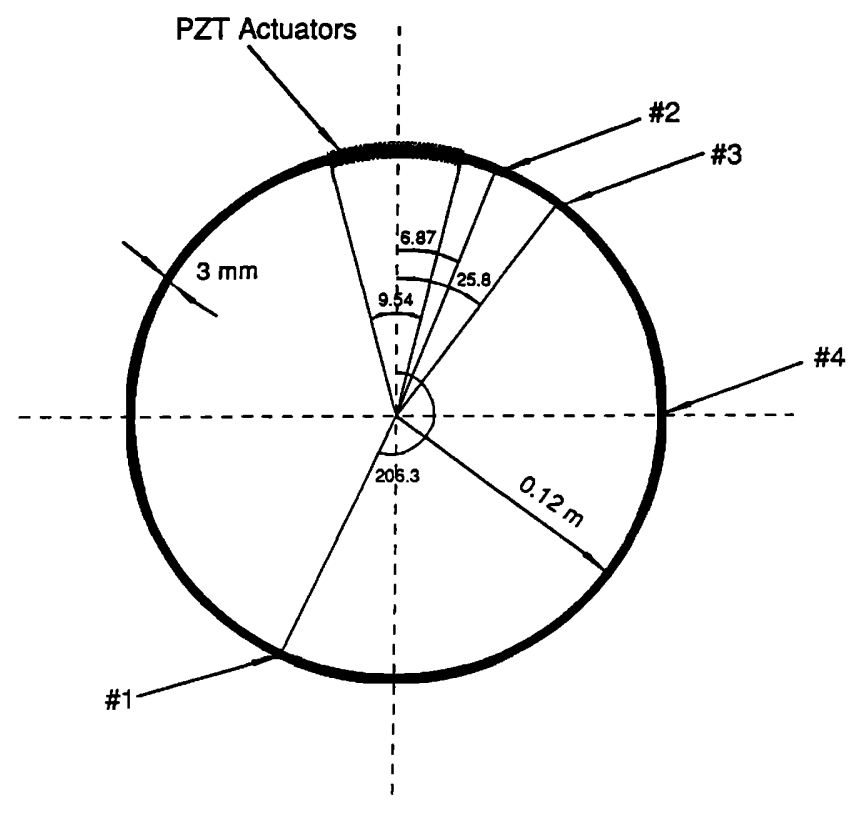

FIG. 3. Dimensions and sensor locations of the circular ring used in the theoretical and experimental investigation.

$$
\bar{w}=\sum_{n=1}^{\infty} \bar{W}_{n} \psi_{n}
$$

and

$$
\bar{v}=\sum_{n=1}^{\infty} \bar{V}_{n} \phi_{n} .
$$

The true structural response under a frequency-dependent actuator excitation can then be obtained by multiplying the transmitting force (or moment) with the above frequency response functions [Eqs. (28) and (29)].

\section{THEORETICAL AND EXPERIMENTAL RESULTS}

Both experimental and theoretical analyses have been conducted to demonstrate the accuracy and utility of the impedance modeling technique. The results from the static approach have also been presented to illustrate the difference between the two approaches.

Figure 3 shows the aluminum ring with bonded PZT actuators used in the experiments and the theoretical analy-

TABLE I. Material properties and dimension of the PZT actuators and aluminum ring.

\begin{tabular}{lll}
\hline \hline & $\begin{array}{c}\text { Aluminum } \\
\text { ring }\end{array}$ & $\begin{array}{l}\text { PZT } \\
\text { actuator }\end{array}$ \\
\hline Young's modulus, Pa & $60 \times 10^{9}$ & $63 \times 10^{9}$ \\
Density, kg/m & 2647 & 7600 \\
Loss factor & 0.005 & 0.01 \\
Piezo. Coef., m/v & N/A & $-166 \times 10^{-12}$ \\
Radius (length), cm & 12.48 & 2.54 \\
Width, cm & 2.54 & 2 \\
Thickness, mm & 3 & 0.23 \\
\hline \hline
\end{tabular}

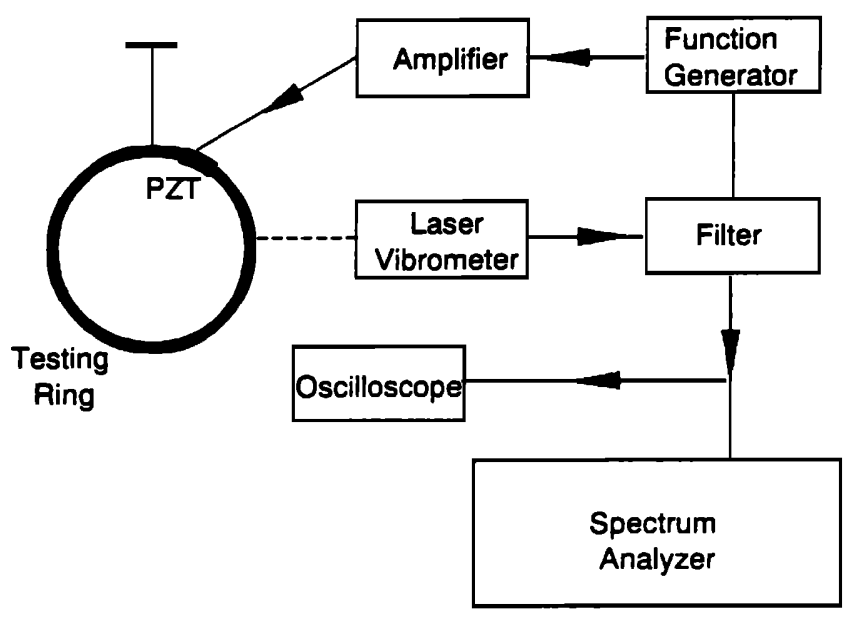

FIG. 4. Experimental setup in the measurement of the ring velocity response. The ring is hung with a fishing line.

sis. The material properties and dimensions of the PZT actuator and the aluminum ring are listed in Table $I$.

The velocity responses of point $\# 1, \# 2, \# 3$, and $\# 4$, as shown in Fig. 3, are calculated using the impedance and static approaches. The ring was hung with a fishing line in the experiment. A laser Doppler measurement system was utilized to measure the velocity response of the ring with bonded PZT actuators. A schematic illustration of the experimental setup is depicted in Fig. 4. The measured and predicted responses of point \#2 and \#4 are shown in Figs. 5 and 6 , respectively.

\section{DISCUSSION}

The predicted and measured dynamic responses of point \#2 are shown in Fig. 5. The static approach provides reasonably accurate results given by the dash-dotted line. The dashed line from the impedance model agrees well with the experimental results (solid line).

Figure 6 shows the measured and predicted responses at point \#4. The experiment results indicate some activities around the second and fourth resonant frequencies, which are

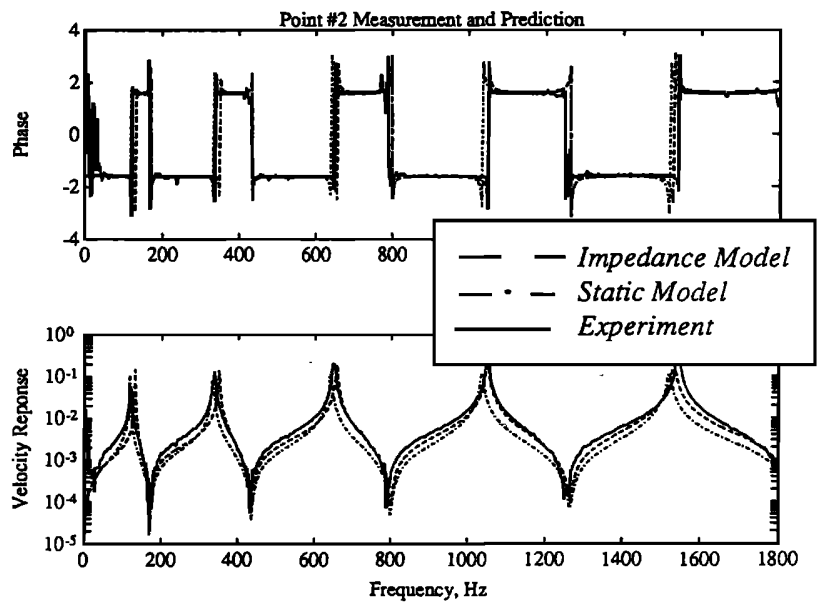

FIG. 5. Measured and predicted velocity and phase response at sensor location \#2.

Rossi et al.: Dynamic analysis of piezoelectric actuators 


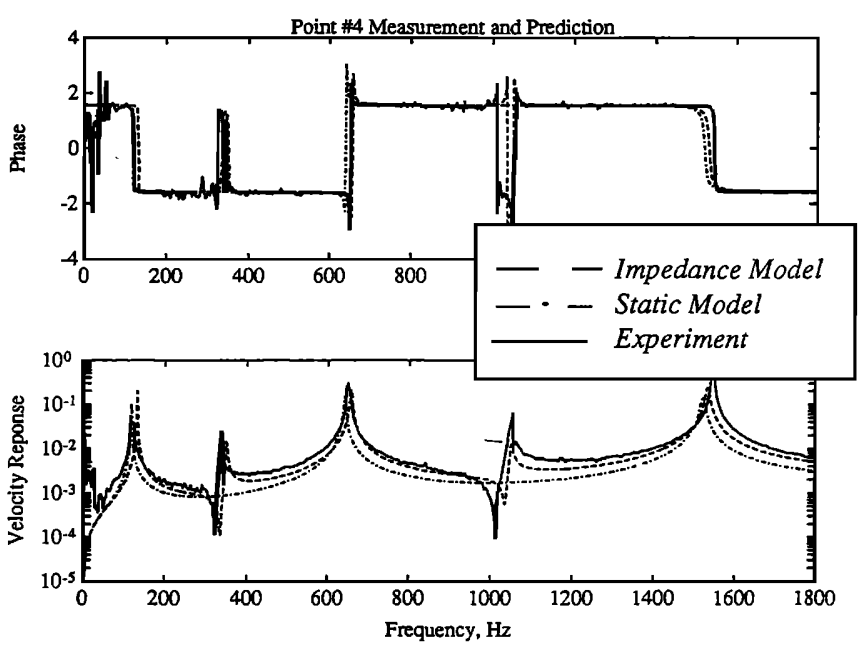

FIG. 6. Measured and predicted velocity and phase response at sensor location \#4.

captured by the impedance model. The static model, in this case, does not even predict the response profile around these two resonant frequencies correctly. This is because the static approach does not include actuator stiffening and mass loading effects. Point \#4 is the nodal line for the second and fourth mode of the ring itself (without PZT actuators). However, once the PZT actuators are bonded to the ring, point \#4 is no longer the nodal line for the new mechanical system (ring and actuators) because of the actuator mass loading and stiffening. The response predicted by the static approach is the frequency response function of the ring itself, while the experimental measurement is the response of the ring and PZT. In this example, the bonding of the actuators may create as much as $45 \%$ stiffening within the ring section where the actuators are bonded.

The results in Fig. 6 may be further explained from the perspective of impedance matching and frequency-dependent actuator excitation. Figure 7 shows the structural and actuator impedance, as well as the stress at the edge of the actuator (the transmitting force per actuator cross-sectional area). This figure demonstrates that the physical essence of the dy-

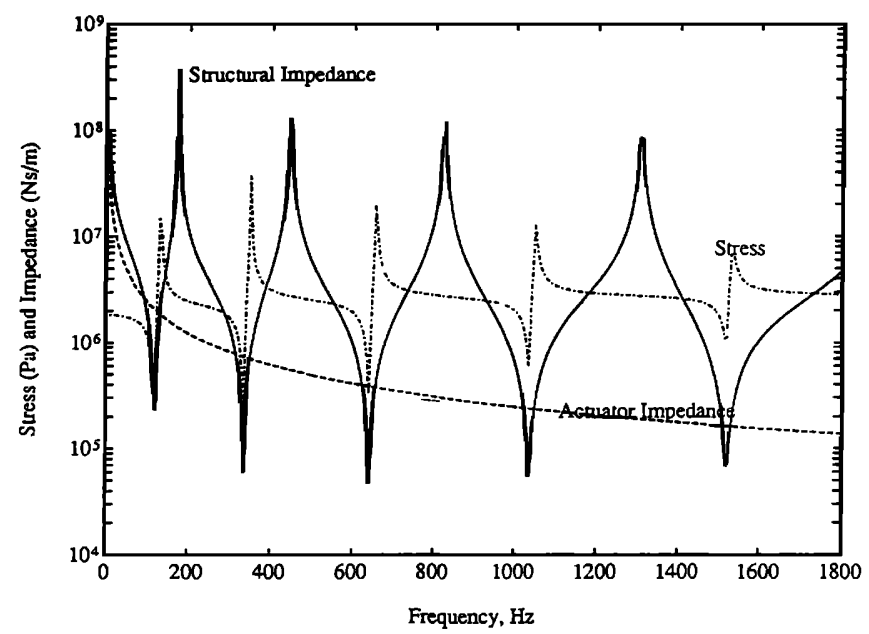

FIG. 7. Structural (solid line) and actuator (dashed line) mechanical impedance, as well as the transmitting force (per actuator cross-sectional area). namic interaction between the integrated induced strain actuators, such as PZT in this case, lies in the match of structural and actuator impedance. At the resonance of the ring (valleys of the structural impedance curve), the actuator transmitting force is at its minimum. The actuator force output reaches a maximum when the actuator impedance matches the structural impedance (complex conjugate). Because the actuators in this case always stiffen the ring, the force output of the actuator demonstrates a repeated valleypeak pattern around each resonant frequency of the ring. It is very clear from this result that the actuator excitation is frequency dependent, which results in the same valley-peak pattern around the second and fourth resonant frequencies for the velocity response of the ring.

The static approach, compared to the impedance method, is simple and can sometimes provide acceptable dynamic response prediction (Clark and Fuller, 1991) if the actuator impedance is much smaller than the structural impedance. The results shown in Fig. 6 also indicate the variation of mode shapes of the ring because of the mass loading and stiffening from the actuators. The impedance model discussed in this paper is more accurate because it considers the stiffening and circumferential mass loading effect (radial direction mass loading effect was not considered) in the analysis. In general, the static model may provide a satisfactory dynamic prediction provided that the actuator is much smaller than the host structure. However, the impedance model can not only provide more accurate results but also reflect the physical essence of the actuator/structure interaction.

\section{CONCLUSION}

The impedance modeling technique developed by Liang et al. (1993) has been utilized to study the dynamic response of circular ring structures. Experiments have been conducted to validate the impedance model. Both experimental and theoretical results indicate that the impedance modeling technique cannot only reflect the physical essence of the dynamic interaction between the integrated induced strain actuators and the host structures, but may also more accurately predict dynamic response than the conventional static approach.

\section{ACKNOWLEDGMENTS}

This work was performed while the first author was a visiting graduate student at the Center for Intelligent Material Systems and Structures. The first author would like to express her gratitude to her adviser Professor Paolo Santini of the Aerospace Department of The University of Rome for giving her the opportunity to study at CIMSS. The study visit was made possible by the fellowship program of ALENIA (Italy). The authors would like to acknowledge the funding support of the Office of Naval Research, Grant ONR 0001492-J-1170, Dr. K. Ng, Program Monitor. The authors would also like to thank Mr. F. P. Sun for his efforts in the experimental work. 
Clark, R. L., Jr., and Fuller, C. R. (1991). "Characterization of Multiple Piezoelectric Actuators for Structural Excitation,” J. Acoust. Soc. Am. 90 (1), 346-357.

Crawley, E. F., and Luis, J. D. (1987). "Use of Piezoelectric Actuators as Elements of Intelligent Structures," AIAA J. 25, 1373-1385.

Dimitriadis, E. K., Fuller, C. R., and Rogers, C. A. (1989). "Piezoelectric Actuators for Distributed Noise and Vibration Excitation of Thin Plates," 8th ASME Conference on Failure, Prevention, Reliability and Stress Analysis, Montreal.
Leissa, A. W. (1973). "Vibration of Shells," NASA Rep.

Liang, C., Sun, F. P., and Rogers, C. A. (1993). "An Impedance Method for the Dynamic Analysis of Active Material Systems," Proceedings, 34th SDM Conference, La Jolla, CA, April 19-22, pp. 3587-3599. Also in ASME J. Vib. Acoust. 116 (1), 120-128 (1994).

Lin, M., and Rogers, C. A. (1993). "Modeling of the Actuation Mechanism in a Beam Structure with Induced Strain Actuators," Proceedings, 34th SDM Conference, La Jolla, CA, 19-21 April, pp. 3608-3617.

Soedel, W. (1981). Vibrations of Shells and Plates (Dekker, New York). 